\title{
The Role of Dexmedetomidine as an Additive to Intravenous Regional Anesthesia: A Systematic Review and Meta-analysis
}

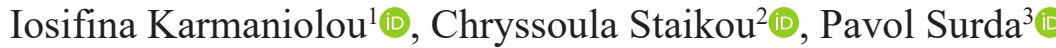

\author{
'Department of Anaesthesia, Guy's and St Thomas' NHS Foundation Trust, London, UK \\ ${ }^{2}$ Department of Anaesthesia, Aretaieio Hospital, National and Kapodistrian University of Athens, Athens, Greece \\ ${ }^{3}$ Department of ENT (ear, nose, and throat), Guy's and St Thomas' NHS Foundation Trust, London, UK
}

Background: Intravenous Regional Anesthesia (IVRA) is a reliable and cost-effective anesthetic method for minor surgical procedures to the extremities. Limitations of this block include tourniquet discomfort, short duration of anesthesia, and absence of postoperative analgesia. Dexmedetomidine has been used as an adjuvant to minimize these negative characteristics with inconclusive results.

Aim: To perform a systematic review of the existing evidence on the role of dexmedetomidine as an additive to intravenous regional anesthesia in upper limb surgery.

Study Design: Systematic Review and Meta-analysis.

Methods: The databases searched were MEDLINE, Embase, PubMed, CINAHL, Cochrane Database of Systematic Reviews, Cochrane Central Register of Controlled Trials databases, and clinicaltrials. gov (1990-2019). Seven randomized controlled trials (RCTs) were included. We analyzed the duration of analgesia, onset time of sensory and motor block, intraoperative tourniquet pain scores, the incidence of tourniquet pain, need for rescue analgesia, intraoperative rescue analgesia consumption, sedation scores, postoperative pain scores, and anesthesia quality.

Results: Intraoperative analgesia duration (MD $11.08 \mathrm{~min}$; 95\% CI $5.42,16.75 ; P<.0001)$ was longer and sensory block onset time (MD $-2.10 \mathrm{~min} ; 95 \% \mathrm{CI}-3.345,-0.86 ; P=.00009)$ was shorter in the dexmedetomidine group. Anesthesia quality scores (MD 0.58; 95\% CI $0.47,0.70 ; P<.00001$ ) and postoperative sedation scores (MD 1.03; $95 \%$ CI $0.88,1.24 ; P<.00001)$ were significantly higher. There was a significant reduction in intraoperative rescue analgesia consumption (MD $-19.70 \mathrm{mg}$; 95\% CI $-24.15,-15.26 ; P<.00001)$ in the dexmedetomidine group. The risk of tourniquet pain as well as postoperative pain scores were lower in favor of dexmedetomidine. The addition of dexmedetomidine to IVRA slightly increased the risk of sedation. A limitation of our study is that some of the interesting outcomes derive from a small number of RCTs.

Conclusion: The addition of dexmedetomidine to IVRA ameliorates the block's characteristics and carries a low risk of potential side effects.

\section{INTRODUCTION}

Intravenous regional anesthesia (IVRA) is known to be a simple, safe, and cost-effective anesthetic method for minor surgical procedures to distal extremities. ${ }^{1}$ Its main drawbacks include short duration of anesthesia, tourniquet discomfort, and lack of postoperative analgesia. ${ }^{2-4}$ Among the adjuvants that have been used to minimize these negative characteristics of IVRA, and dexmedetomidine has been studied in recent years. ${ }^{4,5}$

Dexmedetomidine is a relatively novel, centrally acting alpha- 2 adrenoreceptor agonist; it exhibits high selectivity for alpha-2 receptors, with an $\alpha 2: \alpha 1$ affinity ratio of 1600:1., ${ }^{4,6}$ It produces anxiolysis, sedation, and analgesia, but minimal respiratory depression. ${ }^{4,6}$ Dexmedetomidine has been successfully used as an adjunct in various peripheral nerve blocks. ${ }^{7}$ Its most common side effects are hypotension and bradycardia. ${ }^{7}$

Many investigators have examined the effect of dexmedetomidine as an additive to local anesthetics (LAs) for IVRA, but the results have been so far inconclusive. In the present systematic review and meta-analysis of randomized controlled trials (RCTs), we investigated the efficacy and safety of dexmedetomidine when this is used in combination with LAs for IVRA. Specifically, our aim was to determine the impact of dexmedetomidine on the quality and

Address for Correspondence: Iosifina Karmaniolou, Department of Anaesthesia, Guy's and St Thomas' NHS Foundation Trust, London, UK

e-mail: zozefina_k@hotmail.com

Received: August 11, 2020 Accepted: January 21, 2021 •DOI: 10.5152/balkanmedj.2021.20076

Available at www.balkanmedicaljournal.org

ORCID iDs of the authors: I.K. 0000-0003-2492-6396; C.S. 0000-0001-7336-0443; P.S. 0000-0003-1453-0179.

Cite this article as:

Karmaniolou I, Staikou C, Surda P. The role of dexmedetomidine as an additive to intravenous regional anesthesia: A systematic review and meta-analysis. Balkan Med J. 2021; 38(3):156-164

Copyright@Author(s) - Available online at http://balkanmedicaljournal.org/ 
duration of intraoperative and postoperative analgesia, analgesic drug consumption, and sensory and motor block characteristics. We also assessed the risk of sedation and other adverse effects associated with the administration of dexmedetomidine.

\section{MATERIAL AND METHODS}

Data Sources and Literature Search

We used and adhered to the Preferred Reporting Items for Systematic Reviews and Meta-Analyses (PRISMA) guidelines for the preparation of our study. The databases were searched in March 2019 for RCTs in the English language published between January 1990 and March 2019. We used the Ovid portal to look at MEDLINE, Embase, PubMed, CINAHL, Cochrane Database of Systematic Reviews, Cochrane Central Register of Controlled Trials databases, and clinicaltrials.gov. MeSH and text words were combined using "AND" to generate 2 subsets of citations, comprising studies of dexmedetomidine and the second comprising adjuvant, intravenous regional anesthesia, and Bier block. The references of all the articles found were also manually searched to find any relevant articles not identified through our electronic search. The searches were independently conducted by 2 authors (I.K. and C.S.) and were also carried out just before the final analyses with an aim to capture any recently published articles.

\section{Study Selection}

Data selection and extraction were carried out by 2 authors (I.K. and C.S.) in accordance with the criteria described above. Initially, we examined the titles and abstracts revealed following the electronic searches, and for those abstracts that fulfilled the predefined criteria, the full manuscripts were obtained. For the final decision on inclusion or exclusion, the full manuscripts were thoroughly examined. In the case of manuscripts published twice, the most recent version was chosen.

\section{Data Extraction}

Two reviewers (I.K. and C.S.) completed data extraction. The quality of RCTs was independently reviewed by the same 2 authors (I.K. and C.S.) using the Cochrane Collaboration Risk of Bias tool. Each RCT was scored by consensus; inconsistencies between these 2 reviewers were resolved through discussion with the third reviewer (P.S.) until a consensus was reached. For some of the studies, additional data were deemed necessary. In this case, an email request was sent to the corresponding author of the manuscript. If there was no response during the course of 2 weeks, a second email request was sent.

\section{Data Synthesis}

We included RCTs published from January 1990 until March 2019, which assessed the use of dexmedetomidine as an additive to LAs for IVRA in minor upper limb surgery. In these studies, the dexmedetomidine/intervention group should be compared with a control/placebo group. Trials were excluded if dexmedetomidine was administered neuraxially or perineurally as part of peripheral nerve blocks or intravenously for sedation purposes. RCTs in children were also excluded. Only trials published in English were included in our study. Finally, only studies that had received - and reported-ethical approval from the local/hospital committee or the institutional review board were considered appropriate for the present review.

\section{Outcomes Assessed}

For each study, extracted data included: first author's name, publication year, type of surgery, overall sample size, number of patients in each study group, details about the IVRA technique (LA: type and dose, dexmedetomidine dose), and the perioperative analgesic regimens used. The main outcomes assessed were duration of analgesia, sensory and motor block onset times, intraoperative tourniquet pain scores, the incidence of tourniquet pain, need for rescue analgesia, intraoperative rescue analgesia consumption (measured as micrograms of fentanyl given to a patient during surgery), sedation scores, postoperative pain scores, and quality of anesthesia. Dexmedetomidine-related side effects, namely hypotension, bradycardia, respiratory depression (manifested as desaturation), and sedation, were also sought.

Sensory and motor block duration (in min) was defined as completing LA admixture injection until full recovery from each block. Onset time was defined as the time (in min) from completion of LA injection to achieve the entire sensory and motor block. Duration of analgesia was defined as the time (in min) from cuff inflation to complain about tourniquet pain. Intraoperative and postoperative pain intensity was recorded as VAS scores (Visual Analogue Scale, VAS; $0=$ no pain, $10=$ worst pain imaginable). Sedation level was assessed by the use of Ramsay sedation scale (scores 1-6) as follows: score 1: anxiety or restlessness or both, score 2: patient cooperative, orientated and tranquil, score 3: response to commands, score 4: quick response to stimulus, score 5: sluggish response to stimulus, and score 6 : no response to the stimulus. ${ }^{8}$

\section{Risk of Bias Assessment}

The studies were examined regarding their methodological quality and risk of bias. The following were assessed: randomization method, allocation sequence concealment, blinding of participants and assessors, incomplete or selective outcome data reporting, and other possible sources of bias. The Cochrane "Risk of bias" tool in RevMan 5 was used to describe each domain as it was presented in the study and judgment assignment regarding the respective risk (i.e., high, low, or unclear risk of bias). We give the relevant information in a "risk of bias" summary.

\section{Statistical Analyses}

This work was performed in line with the recommendations of the Cochrane Collaboration and the PRISMA Statement. ${ }^{9,10}$ The author P.S. summarized dichotomous outcome data from each study in $2 \times 2$ tables. The results were pooled using a fixed or random-effect model, as needed, ${ }^{10}$ and expressed as risk ratios (RR). Analysis of continuous variables was performed using mean differences (MD), with $95 \%$ CIs. $^{10}$ Since the studies assessed the same outcome but measured it in various ways, we used standardized mean difference as a summary statistic to standardize the results to a uniform scale before combining them. 
The I2 statistic was used to quantify the heterogeneity of exposure effects across the studies. An I2 value greater than 50\% suggested substantial heterogeneity, and a random-effect model was used. A chi-squared test for heterogeneity was also carried out. The $P$ values are presented.

If only medians were provided, then they were used as estimates of means. When standard deviations (SD) were not available, they were then calculated from the standard error of the mean, $95 \% \mathrm{CI}$, and $t$ value or interquartile range. If trials gave only ranges, then the SD was estimated from the formula: total range/4. The RevMan 5 software (Revman 5.3; Cochrane Library, Oxford, England) was used for the statistical analyses.

\section{RESULTS}

Of the 115 studies retrieved, 7 satisfied the criteria to be included in the analysis (Figure 1). Another 2 studies were excluded because they did not have a control/placebo group..$^{11,12}$ Data from 318 patients were analyzed, in which 159 patients received dexmedetomidine and 159 placebo. Three of the selected trials had a third arm which was not used in the analysis. ${ }^{13-15}$ The included studies are presented in Table 1. The risk of bias is shown in Figure 2.

Intraoperative analgesia, addressed in 4 studies, was significantly longer in dexmedetomidine group (MD $11.08 \mathrm{~min}$; 95\% CI 5.42, 16.75; $P<.0001)^{13,15-17}$ (Figure 3A), even for different LAs and doses of dexmedetomidine ${ }^{13,15-17}$ (Figure $3 \mathrm{~B}$ and $\mathrm{C}$ ).

Of the 7 studies comparing the onset time of the sensory block, Kol et al. presented their results only as a chart and were not

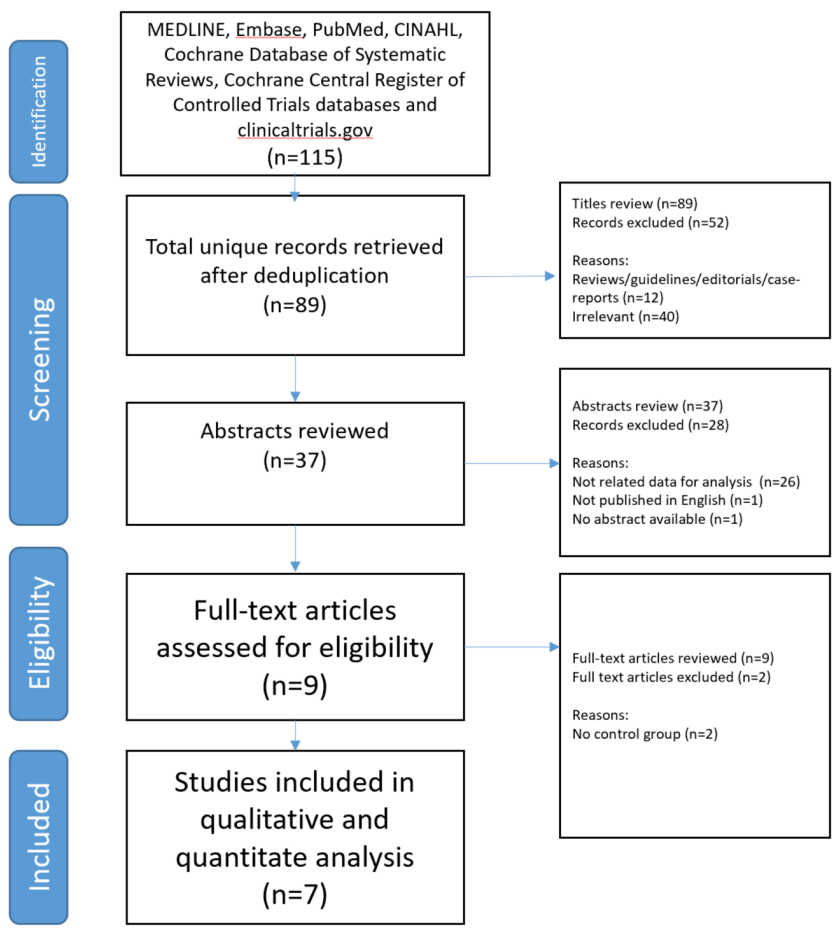

FIG. 1. Consort diagram—study selection process. included in the meta-analysis. ${ }^{13-19}$ Sensory block onset was significantly shorter in dexmedetomidine group (MD $-2.10 \mathrm{~min} ; 95 \%$ $\mathrm{CI}-3.345,-0.86 ; P=.00009$ ) (Figure $4 \mathrm{~A}-\mathrm{C}$ ). No difference was found in motor block onset time ${ }^{13-19}$ (Figure 5A-C). Surgery times did not differ between dexmedetomidine and control groups in the various studies. Surgery times varied between $32 \pm 10.9$ minimum and $53 \pm 14.0$ min maximum in the studies.

Lower Risk Ratio for tourniquet pain was found in dexmedetomidine group (RR 0.38; 95\% CI 0.23, 0.62; $P<.0001){ }^{15,16}$ Overall pain scores were reported on Numerical $0-10^{14,17,19}$ or VAS $0-100$ scales. ${ }^{13}$ Meta-analysis was not possible due to missing values. Differences were observed at 5, 10, 15, 20, 30, and $40 \mathrm{~min}$ in favor of dexmedetomidine by Memis et al. and Subramanya et al. ${ }^{17,19}$ Mizrak et al. did not report a significant difference in pain scores measured every 15 min throughout the procedure. ${ }^{14}$ Dexmedetomidine was associated with a significantly lower incidence of the need for rescue analgesia, as addressed by one study. ${ }^{17}$ Intraoperative rescue analgesia consumption was lower in dexmedetomidine group (MD - $19.70 \mathrm{mg} ; 95 \% \mathrm{CI}$ $-24.15,-15.26 ; P<.00001)^{13,16-19}$ (Figure 6A). This finding was not affected by the type or dose of LA (Figure 6B and C). ${ }^{13,15-17,19}$

Two studies compared the Ramsay sedation scores (1-6 scale). ${ }^{14,15}$ Scores were higher in dexmedetomidine group (MD 1.03; 95\% CI $0.88,1.24 ; P<.00001$. I2 was $48 \%$, therefore fixed effect model was used $\left(\chi^{2}=1.91, P=.17\right)$.

Postoperative pain scores (VAS) were assessed in 2 studies; Esmaoglou et al. used a 0-10 scale, whereas Kol et al. used a $0-100$ scale. $^{13,18}$ We converted all scores to a $0-10$ VAS scale for the purpose of data analysis. We estimated the mean and standard deviation when not reported. ${ }^{13,20}$ Pain scores were significantly lower in dexmedetomidine group, $30 \mathrm{~min}(\mathrm{MD}-1.47 ; 95 \% \mathrm{CI}$ $-2.33,-0.62 ; P=.0007)$ and $2 \mathrm{~h}$ after surgery (MD $-2.93 ; 95 \%$ CI $-4.64,-1.22 ; P=.0007)$.

The quality of anesthesia was assessed by the anesthesiologist in 5 studies. ${ }^{13,15,17-19}$ Kumar et al. and Subramanya et al. reported better quality of anesthesia with dexmedetomidine, ${ }^{15,19}$ but their data were not homogeneous with the rest and were not pooled. The other 3 investigators used the same 1-4 scale, with 4 being the best and 1 being the lowest overall quality of anesthesia. ${ }^{13,17,18}$ They also concluded that anesthesia quality scores were significantly higher with dexmedetomidine (Figure 7A and B).

Regarding adverse events, 5 out of 159 patients who received dexmedetomidine developed bradycardia which required no treatment. ${ }^{16,19}$ Also, Kumar et al. reported that 1 of the 24 patients in the dexmedetomidine group complained of dizziness. ${ }^{15}$ Hypotension or respiratory depression were not observed.

\section{DISCUSSION}

The present meta-analysis indicates that the addition of dexmedetomidine as an adjuvant for IVRA improves block characteristics. It prolongs intraoperative analgesia, while reducing the incidence of tourniquet discomfort, the intensity of intraoperative 


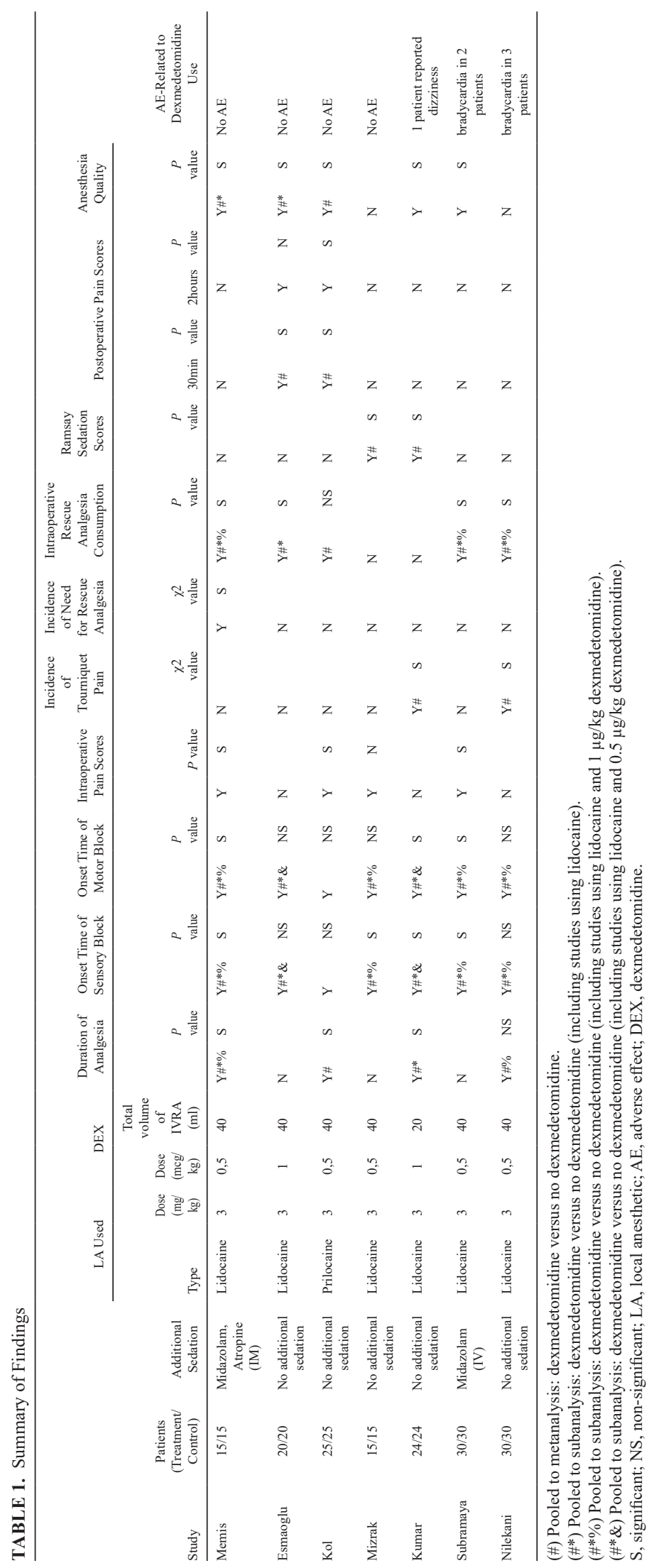




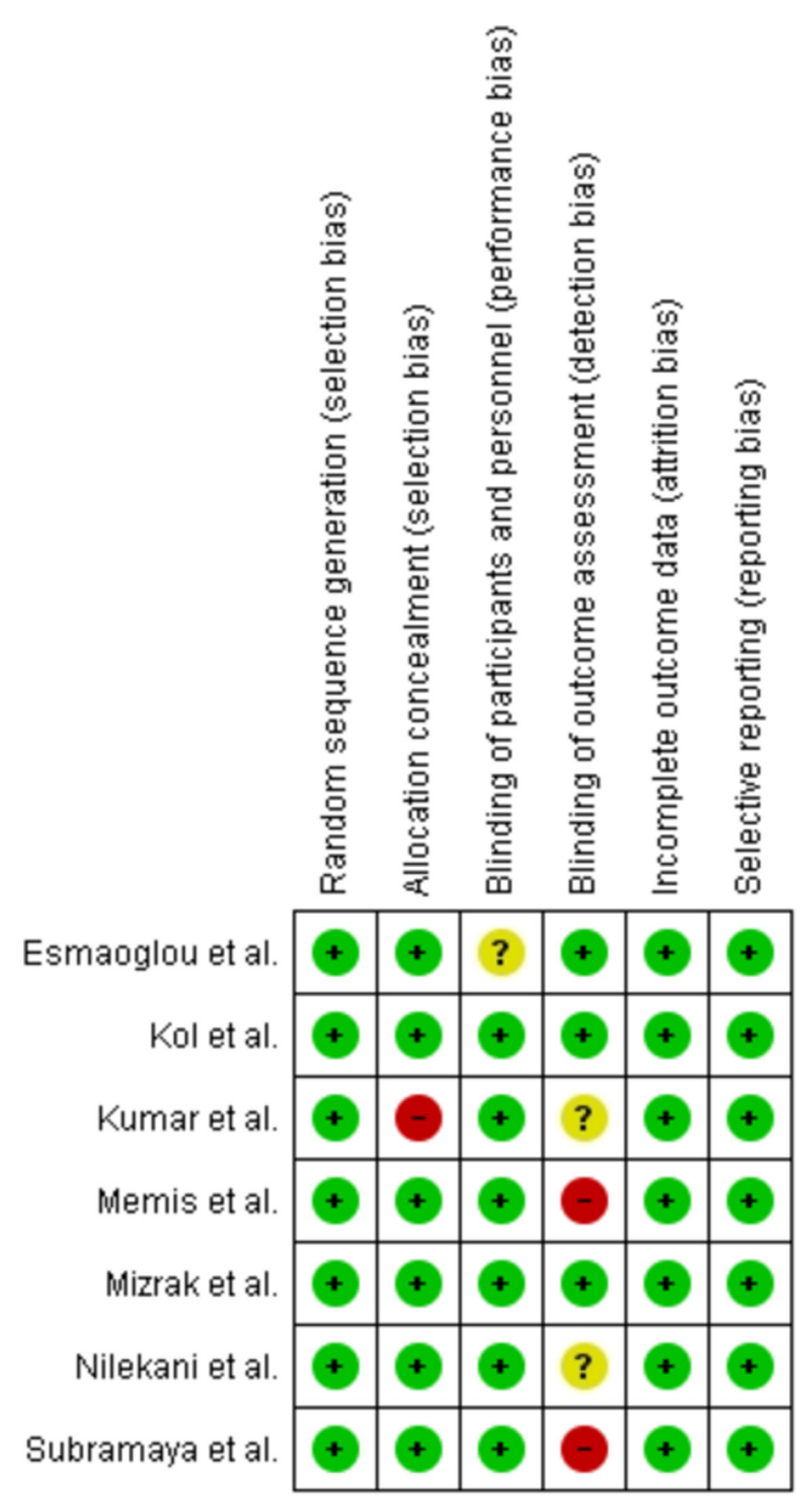

FIG. 2. Risk of bias summary.

pain, and consumption of rescue analgesics. It increases the duration of both sensory and motor blockade and accelerates the onset of sensory block. Finally, it is associated with improved anesthesia quality and less postoperative pain but higher sedation scores.

Dexmedetomidine was approved by the Food and Drug Administration in 1999, and over time it has been used in anesthesia, intensive care, and perioperative medicine..$^{4-6}$ In regional anesthesia techniques, dexmedetomidine has been administered as an "offlabel" adjuvant to LAs for neuraxial, paravertebral, and peripheral nerve blocks; it is considered a promising agent since it has shown beneficial effects on both central and peripheral nerve blocks, without signs of neurotoxicity. ${ }^{5-7,21-24}$
According to our findings, dexmedetomidine improves the quality of intraoperative analgesia, evident by the significant reduction in the incidence of tourniquet pain, in intraoperative pain scores, and fentanyl consumption, used as rescue analgesia. ${ }^{13,15-19}$ It also prolonged the time of tourniquet tolerance ${ }^{13,15-17}$ significantly; this advantage was shown when dexmedetomidine was added to lidocaine and prilocaine. ${ }^{13}$ No study used ropivacaine, which is per se associated with IVRA prolongation. Thus no assumptions can be made for the effects of dexmedetomidine when combined with LAs of longer duration of action. ${ }^{2}$ The prolongation of intraoperative analgesia by dexmedetomidine may be related to the prolongation of sensory block, as our results showed. Regardless of the mechanism, this effect renders dexmedetomidine a very advantageous adjuvant. One of the main drawbacks of IVRA is its short duration and subsequent development of tourniquet discomfort/ pain. Various adjuvants have been used to overcome these problems, such as opioids, non-steroidal anti-inflammatory drugs, corticosteroids, neuromuscular blockers, ketamine, magnesium, potassium, and $\alpha-2$ agonists..$^{2-4}$ Among them, opioids did not show any clinically significant benefit, while clonidine, ketamine, and lornoxicam had positive effects on analgesia and were also compared with dexmedetomidine. .,3,12,13,15 $^{2}$

Sardesai et al. found dexmedetomidine superior to clonidine as IVRA adjuvant, in terms of quality and total duration of analgesia. ${ }^{12}$ These effects may be associated with the significantly higher a2-receptor specificity (7-8 times) and lipophilicity (about 3.5 times) of dexmedetomidine than clonidine. ${ }^{25}$ Other mechanisms, such as blockade of the hyperpolarization-activated cation current $\left(\mathrm{I}_{\mathrm{h}}\right)$, may also play a role. ${ }^{25,26}$ According to experimental data, perineural dexmedetomidine not only prolongs ropivacaine sensory analgesia, but may itself induce a partial sensory block of short duration. It has been suggested that these effects are mediated via peripheral mechanisms (i.e., $\mathrm{I}_{\mathrm{h}}$ current inhibition) and not through central/systemic actions. ${ }^{26}$

Dexmedetomidine was found similarly efficacious with ketamine and lornoxicam. ${ }^{13,15}$ Kumar et al. found that ketamine was associated with better intraoperative analgesia and delayed onset of tourniquet pain, whereas dexmedetomidine produced more prolonged postoperative analgesia. ${ }^{15}$ The 2 agents were found comparable in terms of postoperative pain intensity. ${ }^{15}$ Likewise, Kol et al. found no significant difference between dexmedetomidine and lornoxicam when combined with prilocaine for IVRA regarding the quality and duration of intraoperative analgesia for tourniquet pain. The duration of postoperative analgesia was also comparable between the 2 adjuvants. ${ }^{13}$

The meta-analysis of 6 studies clearly showed that dexmedetomidine accelerates the onset of sensory block. ${ }^{14-19}$ Importantly, a dose of $0.5 \mu \mathrm{g} / \mathrm{kg}$ is adequate to produce this beneficiary effect. Interestingly, higher doses (i.e., $1 \mu \mathrm{g} / \mathrm{kg}$ ) did not seem to affect the sensory block onset time when the respective studies were analyzed separately. ${ }^{15,18}$ Whereas sensory block onset was shortened by dexmedetomidine, motor block onset was not affected. ${ }^{14-19} \mathrm{~A}$ higher impact of dexmedetomidine on sensory rather than a motor 


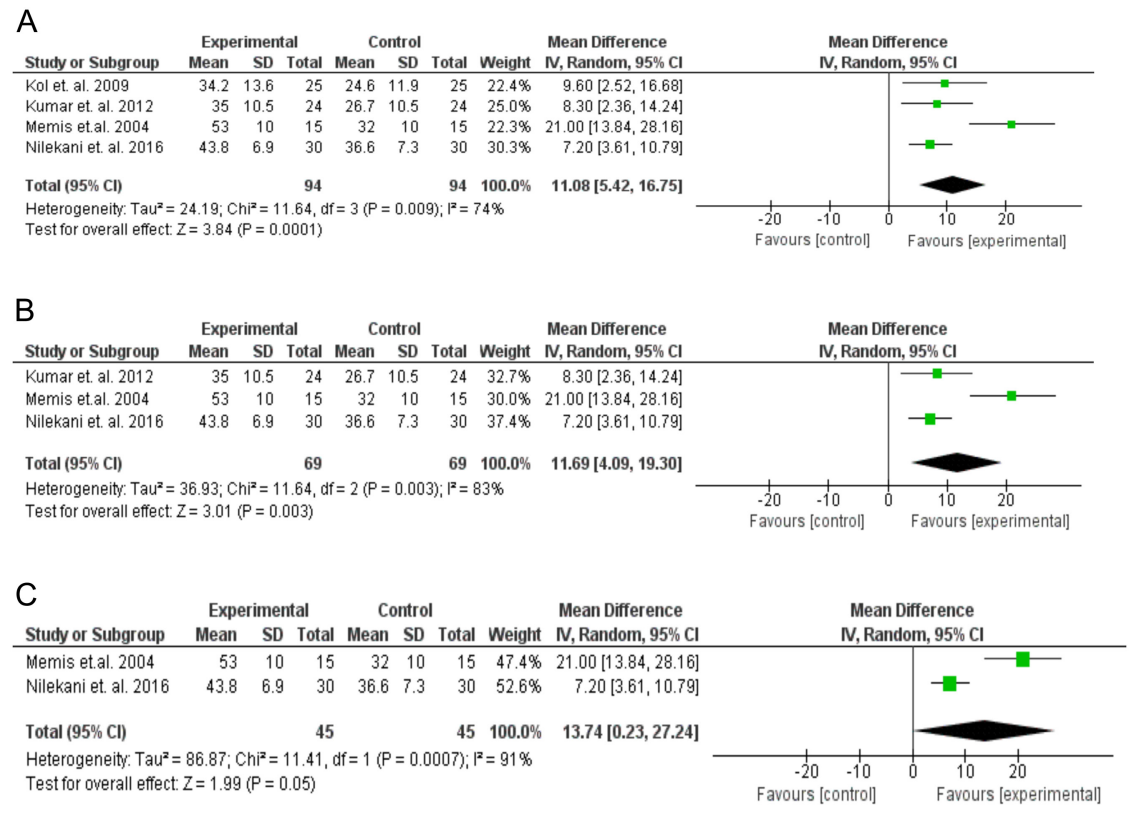

FIG. 3. A-C. Forest plots of comparison-duration of analgesia (min). (A) Forest plot of comparison: dexmedetomidine vs no dexmedetomidine.(B) Subgroup analysis: dexmedetomidine vs no dexmedetomidine (including studies using lidocaine). (C) Subgroup analysis: dexmedetomidine vs no dexmedetomidine (including studies using lidocaine and $0.5 \mathrm{mcg} / \mathrm{kg}$ dexmedetomidine).

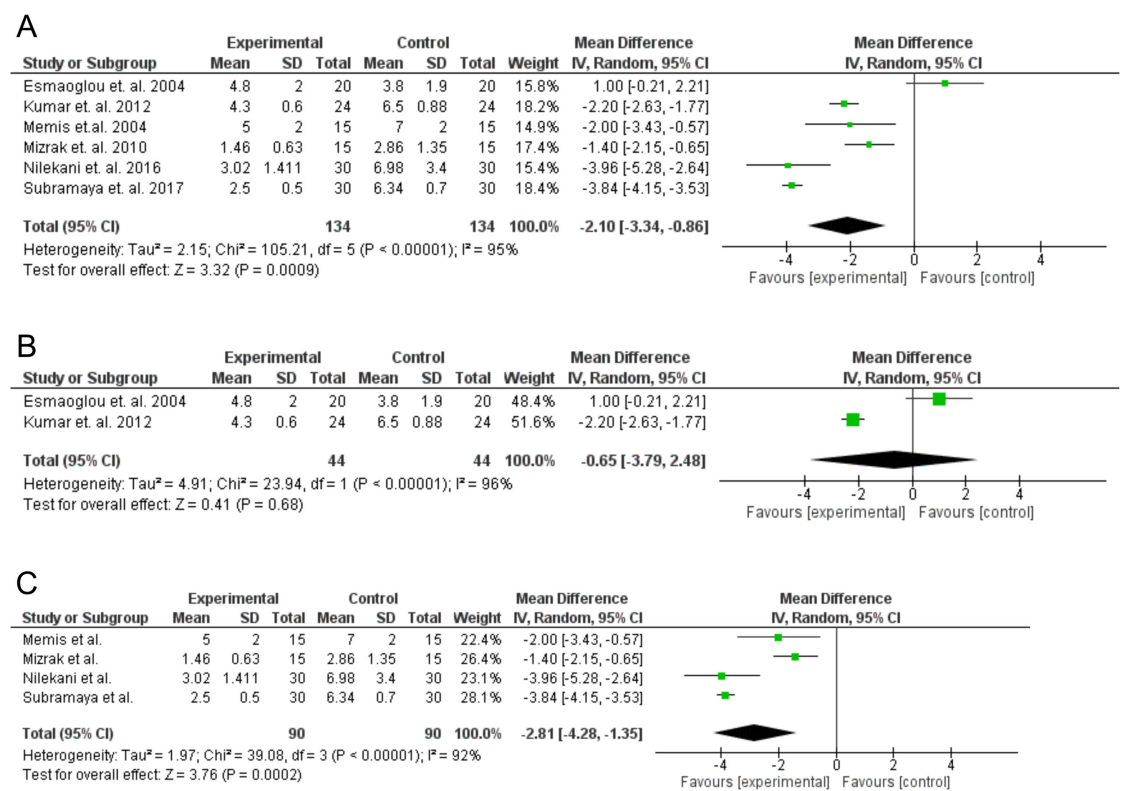

FIG. 4. A-C. Forest plots of comparison - onset time of sensory block (minutes). (A) Forest plot of comparison: dexmedetomidine vs no dexmedetomidine. (B) Subgroup Analysis: dexmedetomidine vs no dexmedetomidine (including studies using lidocaine and $1 \mathrm{mcg} / \mathrm{kg}$ dexmedetomidine). (C) Subgroup Analysis: dexmedetomidine vs no dexmedetomidine (including studies using lidocaine and $0.5 \mathrm{mcg} / \mathrm{kg}$ dexmedetomidine).

block has been previously documented, too; it is possibly associated with a more pronounced inhibitory effect on A $\delta$ and $\mathrm{C}$ fiber action potentials than motor neurons. ${ }^{27-29}$

Dexmedetomidine prolonged intraoperative analgesia, irrespective of the dose used. This finding was consistent with both prilocaine and lidocaine analyses and when a subgroup analysis for lidocaine was only undertaken. Anesthesia quality, documented mainly by the anesthesiologist in the form of a scale, presented substantial heterogeneity, and all studies could not be included. However, analyzed data showed that dexmedetomidine offered a higher quality of anesthesia, which is a significant clinical advantage. 


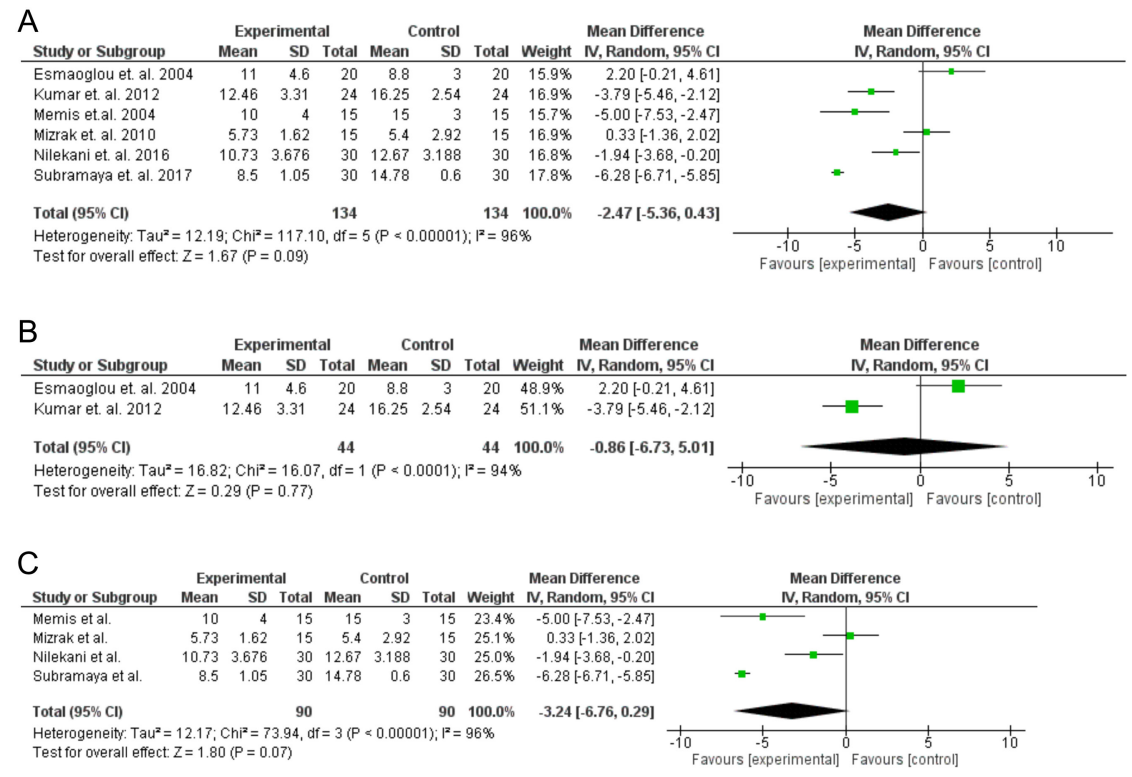

FIG. 5. A-C. Forest plots of comparison - onset time of motor block (minutes). (A) Forest plot of comparison: dexmedetomidine vs no dexmedetomidine. (B) Subgroup Analysis: dexmedetomidine vs no dexmedetomidine (including studies using lidocaine and $1 \mathrm{mcg} / \mathrm{kg}$ dexmedetomidine). (C) Subgroup Analysis: dexmedetomidine vs no dexmedetomidine (including studies using lidocaine and $0.5 \mathrm{mcg} / \mathrm{kg}$ dexmedetomidine).
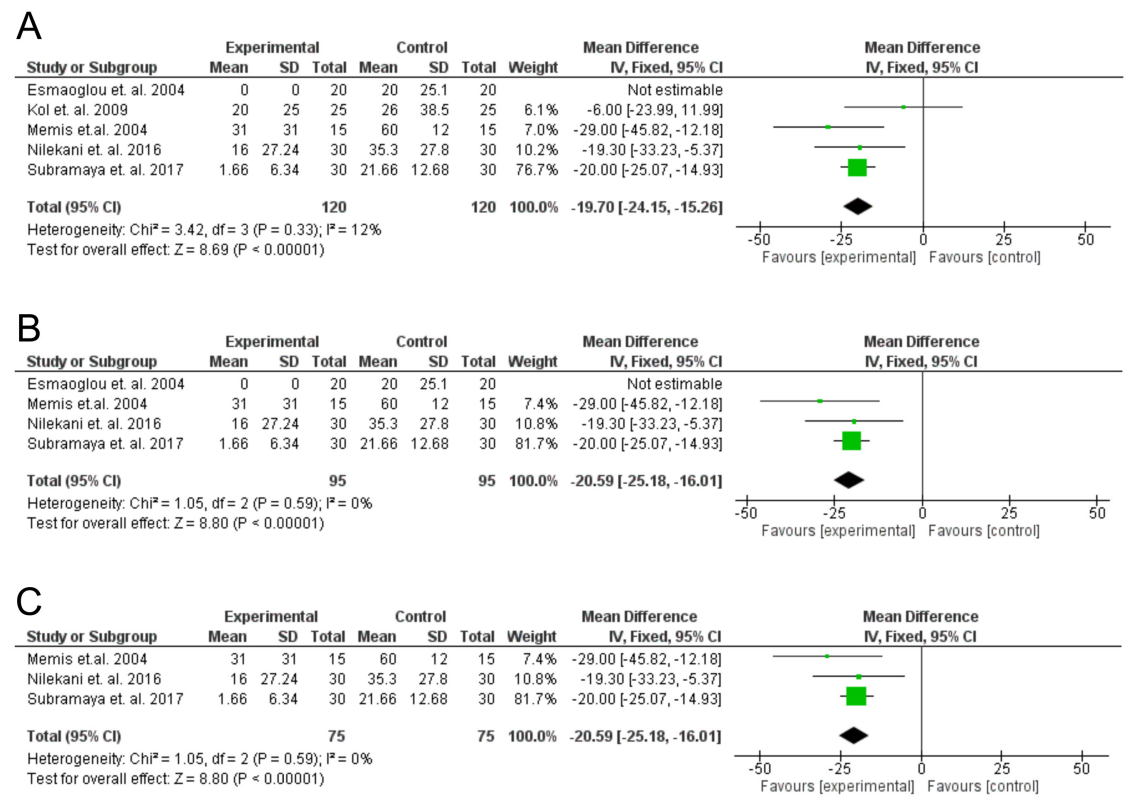

FIG. 6. A-C. Forest plots of comparison - Intraoperative rescue analgesia consumptionmeasured as the amount (mcg) of fentanyl administered intraoperatively. (A) Forest plot of comparison: dexmedetomidine vs no dexmedetomidine. (B) Subgroup Analysis: dexmedetomidine vs no dexmedetomidine (including studies using lidocaine). (C) Subgroup Analysis: dexmedetomidine vs no dexmedetomidine (including studies using lidocaine and $0.5 \mathrm{mcg} / \mathrm{kg}$ dexmedetomidine).

There are, of course, some complications associated with IVRA. These are mainly associated with the inevitable leakage of part of the local anesthetic administered into the circulation. In particular, these mainly include sedation, bradycardia, and hypotension. These can be attributed to accidental or unintentional deflation of the cuff, cuff default, an increase in venous pressure within the occluded extremity to a level higher than that of the cuff, and lastly, via an intact interosseous circulation. Even when the tourniquet is functioning properly, there is still a risk of leakage of the drugs administered in an isolated extremity into the systemic circulation. 

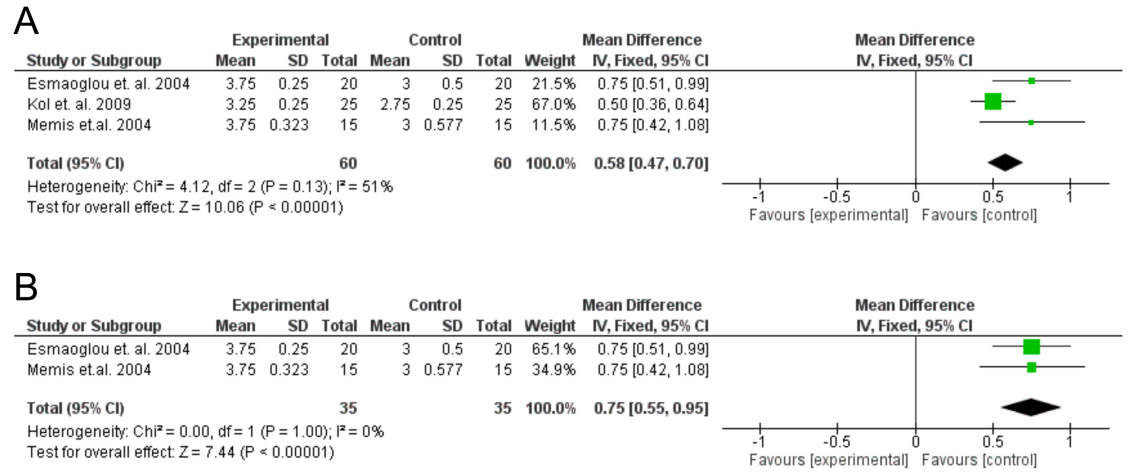

FIG. 7. A-B. Forest plots of comparison—anesthesia quality. (A) Forest plot of comparison: dexmedetomidine vs no dexmedetomidine. (B) Subgroup Analysis: dexmedetomidine vs no dexmedetomidine (including studies using lidocaine).

The incidence of local anesthetic leakage from beneath the tourniquet is almost $100 \%$ for lower limb IVRA and approximately $25 \%$ for an upper extremity block.

In the early postoperative period, sedation was more prominent in dexmedetomidine groups. Still, the mean values of Ramsay scores remained below the level of 3 , which corresponds to a "drowsy patient, responsive to commands". ${ }^{14,15}$ Thus, we consider that the sedation was not too deep, as to be characterized as unwanted or associated with increased risks; a score of 2 to 3 mainly indicates anxiolysis, which is desired in everyday clinical practice, since the patient is calm and responsive, but not asleep (as in scores $\geq 4$ ). The higher Ramsay scores were rather expected, since dexmedetomidine exhibits significant anxiolytic, sedative, and analgesic properties due to its a-2 adrenoreceptor selectivity, especially for the subtypes $\alpha-2 \mathrm{~A}$ and $\alpha-2 \mathrm{C}^{4,6,30}$

Bradycardia was documented, but it was not profound as to require treatment. It is a side-effect of dexmedetomidine due to central sympatholysis and inhibition of noradrenaline release caused via activation of post-synaptic and pre-synaptic $\alpha-2$ adrenoreceptors, respectively ${ }^{4,6}$ Hypotension, which is attributable to the same mechanism, was not recorded.

To our knowledge, there are no previous systematic reviews with which to compare our findings. Our study is subjected to some limitations. Small sample sizes and different doses of dexmedetomidine have caused heterogeneity. In order to overcome this risk, we performed subgroup analyses where data were available. However, this was not possible for all comparisons. Potential bias in the review process regarding the eligibility criteria and data analyses is another limitation of our study. Lastly, publication bias may have impacted our results. Despite these limitations, the present review attempted to bring the existing evidence together, address heterogeneity during the statistical analysis and provide reliable information on the research subject.

The present study was presented as a poster at the 38th Annual ESRA Congress in Bilbao, Spain, September 11-14, 2019.

Ethics Committee Approval: N/A.

Patient Consent for Publication: N/A.
Author Contributions: Design - I.K.; Data Collection and/or Processing - I.K., C.S.; Analysis and/or Interpretation - P.S.; Writing - I.K., C.S., P.S.

Conflicts of Interest: The authors have no conflicts of interest to declare.

Funding: The authors declared that this study had received no financial support.

\section{REFERENCES}

1. Brown EM, McGriff JT, Malinowski RW. Intravenous regional anaesthesia (Bier block): review of 20 years' experience. Can J Anaesth. 1989;36(3 Pt 1):307-310. [CrossRef]

2. Flamer D, Peng PW. Intravenous regional anesthesia: a review of common local anesthetic options and the use of opioids and muscle relaxants as adjuncts. Local Reg Anesth. 2011;4:57-76. [CrossRef]

3. Choyce A, Peng P. A systematic review of adjuncts for intravenous regional anesthesia for surgical procedures. Can J Anaesth. 2002;49(1):32-45. [CrossRef]

4. Ramadhyani U, Park JL, Carollo DS, Waterman RS, Nossaman BD. Dexmedetomidine: clinical application as an adjunct for intravenous regional anesthesia. Anesthesiol Clin. 2010;28(4):709-722. [CrossRef]

5. Marhofer P, Brummett CM. Safety and efficiency of dexmedetomidine as adjuvant to local anesthetics. Curr Opin Anaesthesiol. 2016;29(5):632-637. [CrossRef]

6. Bajwa S, Kulshrestha A. Dexmedetomidine: an adjuvant making large inroads into clinical practice. Ann Med Health Sci Res. 2013;3(4):475-483. [CrossRef]

7. Schnabel A, Reichl SU, Weibel S, et al. Efficacy and safety of dexmedetomidine in peripheral nerve blocks: A meta-analysis and trial sequential analysis. Eur J Anaesthesiol. 2018;35(10):745-758. [CrossRef]

8. Ramsay MA, Savege TM, Simpson BR, Goodwin R. Controlled sedation with alphaxalone-alphadolone. Br Med J. 1974;2(5920):656-659. [CrossRef]

9. Liberati A, Altman DG, Tetzlaff J, et al. The PRISMA statement for reporting systematic reviews and meta-analyses of studies that evaluate healthcare interventions: explanation and elaboration. Br Med J. 2009;339:b2700. [CrossRef]

10. Clarke M, Horton R. Bringing it all together: lancet-cochrane collaborate on systematic reviews. Lancet. 2001;357(9270):1728. [CrossRef]

11. Gupta B, Verma RK, Kumar S, Chaudhary G. Comparison of analgesic efficacy of dexmedetomidine and midazolam as adjuncts to lignocaine for intravenous regional anesthesia. Anesth Essays Res. 2017;11(1):62-66. [CrossRef]

12. Sardesai SP, Patil KN, Sarkar A. Comparison of clonidine and dexmedetomidine as adjuncts to intravenous regional anaesthesia. Indian J Anaesth. 2015;59(11):733-738 [CrossRef]

13. Kol IO, Ozturk H, Kaygusuz K, et al. Addition of dexmedetomidine or lornoxicam to prilocaine in intravenous regional anaesthesia for hand or forearm surgery: a randomized controlled study. Clin Drug Investig. 2009;29(2):121-129. [CrossRef]

14. Mizrak A, Gul R, Erkutlu I, Alptekin M, Oner U. Premedication with dexmedetomidine alone or together with 0.5\% lidocaine for IVRA. J Surg Res. 2010;164(2):242247. [CrossRef]

15. Kumar A, Sharma DK, Datta B. Addition of ketamine or dexmedetomidine to lignocaine in intravenous regional anesthesia: a randomized controlled study. $J$ Anaesthesiol Clin Pharmacol. 2012;28(4):501-504. [CrossRef] 
16. Nilekani E, Menezes Y, D'Souza SA. A study on the efficacy of the addition of low dose dexmedetomidine as an adjuvant to lignocaine in intravenous regional anaesthesia (IVRA). J Clin Diagn Res. 2016;10:UC01-UC05. [CrossRef]

17. Memiş D, Turan A, Karamanlioğlu B, Pamukçu Z, Kurt I. Adding dexmedetomidine to lidocaine for intravenous regional anesthesia. Anesth Analg. 2004;98(3):835-840, table of contents. [CrossRef]

18. Esmaoglu A, Mizrak A, Akin A, Turk Y, Boyaci A. Addition of dexmedetomidine to lidocaine for intravenous regional anaesthesia. Eur J Anaesthesiol. 2005;22(6):447451. [CrossRef]

19. Subramanya V, Kapinigowda ST, Math AT, Chennaiah VB. Dexmedetomidine as an adjuvant for intravenous regional anesthesia in upper limb surgeries. Anesth Essays Res. 2017;11(3):661-664. [CrossRef]

20. Hozo SP, Djulbegovic B, Hozo I. Estimating the mean and variance from the median, range, and the size of a sample. BMC Med Res Methodol. 2005;5:13. [CrossRef]

21. Wu HH, Wang HT, Jin JJ, et al. Does dexmedetomidine as a neuraxial adjuvant facilitate better anesthesia and analgesia? A systematic review and meta-analysis. PLOS ONE. 2014;9(3):e93114. [CrossRef]

22. Wang K, Wang LJ, Yang TJ, et al. Dexmedetomidine combined with local anesthetics in thoracic paravertebral block: a systematic review and meta-analysis of randomized controlled trials. Medicine. 2018;97(46):e13164. [CrossRef]

23. Sun Q, Liu S, Wu H, et al. Dexmedetomidine as an adjuvant to local anesthetics in transversus abdominis plane block: a systematic review and meta-analysis. Clin J Pain. 2019;35(4):375-384. [CrossRef]
24. Shamil E, Rouhani MJ, Basetti S, et al. Role of local anaesthetic nerve block in endoscopic sinus surgery: a systematic review and meta-analysis. Clin Otolaryngol. 2018;43(5):1201-1208. [CrossRef]

25. Kaur M, Singh PM. Current role of dexmedetomidine in clinical anesthesia and intensive care. Anesth Essays Res. 2011;5(2):128-133. [CrossRef]

26. Brummett CM, Amodeo FS, Janda AM, Padda AK, Lydic R. Perineural dexmedetomidine provides an increased duration of analgesia to a thermal stimulus when compared with a systemic control in a rat sciatic nerve block. Reg Anesth Pain Med. 2010;35(5):427-431. [CrossRef]

27. El-Boghdadly K, Brull R, Sehmbi H, Abdallah FW. Perineural dexmedetomidine is more effective than clonidine when added to local anesthetic for supraclavicular brachial plexus block: a systematic review and meta-analysis. Anesth Analg. 2017;124(6):2008-2020. [CrossRef]

28. Abdallah FW, Dwyer T, Chan VWS, et al. IV and perineural dexmedetomidine similarly prolong the duration of analgesia after interscalene brachial plexus block: a randomized, three-arm, triple-masked, placebo-controlled trial. Anesthesiology. 2016;124(3):683-695. [CrossRef]

29. Abdallah FW, Brull R. Facilitatory effects of perineural dexmedetomidine on neuraxial and peripheral nerve block: a systematic review and meta-analysis. $\mathrm{BrJ}$ Anaesth. 2013;110(6):915-925. [CrossRef]

30. Bharti N, Sardana DK, Bala I. The analgesic efficacy of dexmedetomidine as an adjunct to local anesthetics in supraclavicular brachial plexus block: a Randomized Controlled Trial. Anesth Analg. 2015;121(6):1655-1660. [CrossRef] 